\title{
Radialsymmetrischer Scherkraftaufnehmer als Transfernormal
}

\author{
Thomas Kleckers \\ Hottinger Baldwin Messtechnik GmbH \\ Im Tiefen See 45 \\ 64293 Darmstadt \\ +496151803570 \\ Thomas.kleckers@hbm.com
}

Radialsymmetrische Scherkraftaufnehmer sind in der experimentellen Mechanik ein bewährtes und häufig genutztes Messmittel. Neueste Entwicklungen zeigen, dass das Potential dieser Technologie auch den Einsatz als präzises Transfernormal für Referenzkraftmessungen erlaubt- mit hoher Genauigkeit zu vertretbaren Kosten.

\section{Funktionsweise von radialsymmetrischen Scherkraftaufnehmern}

Das Funktionsprinzip von Dehnungsmessstreifen - basierte Kraftaufnehmer ist seit der ersten industriellen Produktion 1956 bewährt und wurde immer weiter entwickelt. Ein metalliischer Federkörper wird in den Kraftfluss eingebaut. Die Einwirkenden Kräfte erzeugen mechanische Spannungen, die iherseits nach dem Hookschen Gesetz Dehnungen nach sich ziehen. In zuvor bestimmten Zonen werden Dehnungsmessstreifen aufgeklebt, die diese Dehnungen in Widerstandsänderungen wandeln. Durch Verschaltung zur so genannten Wheatston'schen Brückenschaltung und externer Spannungsversorgung entsteht ein der eingeleiteten Kraft proportionales Spannungssignal.

Die Besonderheit der symmetrischen Scherkraftaufnehmern liegt in der Kombination der folgenden konstruktiven Details:

- Die eingesetzten Dehnungsmesstreifen basieren auf Chrom - Nickel - Legierungen, die einen höheren K - Faktor (eine höhere Empfindlichkeit) als die meist genutzten Konstantan - DMS auf. Die Empfindlichkeit ist um etwa $10-15 \%$ höher.

- Das Dehnungsfeld im Bereich der DMS - Installation ist ohne Gradienten oder Dehnungsmaxima von einem gleichmäßigen Verlauf. Damit ist es möglich, den gesamten DMS mit dem technisch größten Dehnungsniveau zu belasten, was ein maximale Widerstandsänderung nach sich zieht.

- Die Konstruktion is so ausgelegt, dass die höchsten mechanischen Spannungen im Bereich der DMS vorzufinden sind, alle anderen Bereiche des Kraftaufnehmers sind nur geringen mechanischen Spannungen ausgesetzt.

Aus den oben genannten Konsstruktionsdetails ergeben sich eine Reihe von Vorteilen, die den Erfolg dieses Messprinzipes in Experiment und am Prüfstand erklärt:

- Bereits bei geringer mechanischer Belastung der DMS ein steht ein hohes Ausgangssignal zur Verfügung. Wird der Sensor auf die in der Kraftmesstechnik üblichen $2 \mathrm{mV} / \mathrm{V}$ kalibriert, ergeben sich sehr hohe mechanische Reserven. (Überlasttoleranz, Wechsellastfähigkeit)

- Umbekehrt ermöglicht dieses Messprinzip die Kalibrierung bei höheren Kräften, so dass das Ausgangssignal bis zu $4 \mathrm{mV} / \mathrm{V}$ beträgt. Dies entspricht dem doppelten Wert, den andere Prinzipien bei vergleichbarer Betriebsfestigkeit zur Verfügung stellen. In diesem Fall verkleinert sich die Tolernaz gegenüber Überlasten und die Wechsellastfähigkeit verglichen mit einer 2 mV/N Kalibrierung, jedoch erhöht sich die Genauigkeit beträchtlich.

Es zeigt sich, dass vor allem die dynamischen Anforderungen bei Betriebsfestigkeitsprüfungen und in Prüfständen von radialsymmetrische Scherkraftsensoren seht gut erfüllt wird. 


\section{Einsatz als Transfernormal}

In Experiment und am Prüfstand steigen die Anforderungen an die Genauigkeit der eingesetzten Kraftsensoren und der angeschlossenen Elektronik stetig. Dies hat wirtschaftliche Gründe, da höhere Genauigkeit zu erweiterten Messbereichen und damit zu größerem Einsatzbereich des Prüfstandes führt.

Bei Transfernomalen, also Kraftaufnehmer die dazu dienen, Kalibriermaschinen zu vergleichen und existierende Maschinen über Vergleichsmessungen an eine höheres Normal anzuschließen, kommen zahlreiche Forderungen zu den Anforderungen aus Experiment und Prüfstand hinzu:

- Die Widerholpräzision in verschiedenen Einbaustellungen ist ein Merkmal der maßgeblichen ISO376. Hiermit wird garantiert dass der Einbau zur Kalibrierung ohne großen Einfluss auf das Ergebnis ist.

- Die Grenzen für die Umkehrspanne (Hysterese) sin deutlich enger gesetzt als für die industrielle Anwendung.

- Die Kennlinie des Sensors soll durch eine kubische Approximation sehr gut beschrieben werden können- in industriellen Anwendungen ist die Abweichung von einer Geraden (Linearitätsabweichung) entscheidend

Radialsymmetrische Scherkraftaufnehmer sind Sensoren, die Schraubverbindungen aufweisen, um den eigentlichen Federkörper mit dem unbedingt erforderlichen Fußadapter zu verbinden. Diese Flanschverschraubung ist ein Unterschied zu konventionellen Transfernormalen

(Referenzkraftaufnehmer), die monolithisch konstruiert sind. Schraubverbindungen im Kraftfluss limitieren die Genauigkeit, da kleinste Verschiebungen der verbundenen Konstruktionselemente zueinander geänderte Dehnungszustände im Bereich der DMS verursachen- im Endergebnis sind diese als Hysterese nachweisbar.

\section{Neues Transfernormal}

Erste Referenzkraftaufnehmer nach dem Scherprinzip wurden in den frühen 2000er Jahren zur Marktreife gebracht.

Diese Kraftmessdosen sind für Zug- und Druckbelastungen ausgelegt und erreichen die Klasse 0,5 nach ISO376, das ist die zweitbeste Klassengenauigkeit, die dieser international gültige Standard vorsieht. Die Anfälligkeit des Prinzips gegen Einflüsse der Verschraubung verhinderte eine bessere Klasseneinstufung. Dies gilt bei Zugkräften, wenn auf die Verbindung die zu messende Kraft und die Vorspannung durch die Verschraubung selbst einwirken. Bei Messung von Druckkräften ist dies nicht von Bedeutung.

Letzte Entwicklung ist ein Druckkraftaufnehmer, der die höchste Genauigkeitsklasse 00 nach ISO376 erreicht. Um dieses Ziel konstruktiv zu erreichen, waren verschiedenen Maßnahmen notwendig:

- Die Biegesteifigkeit des Sensors musste zurückgenommen werden, um den Einfluss der Eigenschaften von Kalibriermaschinen auf das Ergebnis zu verringern

- Beschränkung auf Druckkräfte, um den Einfluss der Verschraubung zu minimieren.

Mit einer Neukonstruktion der Lasteinleitung konnten die Ziele erreicht werden Hierbei ist eine Nut in den Fußadapter eingebracht, so dass die Biegemomentsteifigkeit gezielt zurückgenommen wird. Eventuell während der Kalibrierung auftretende Momente werden somit nicht mehr in den Federkörper geleitet und sind ohne Einfluss auf das Ergebnis. Durch verbesserte Ebenheit konnte der Kraftschluss zwischen Fußadapter und Messkörper verbessert werden. 


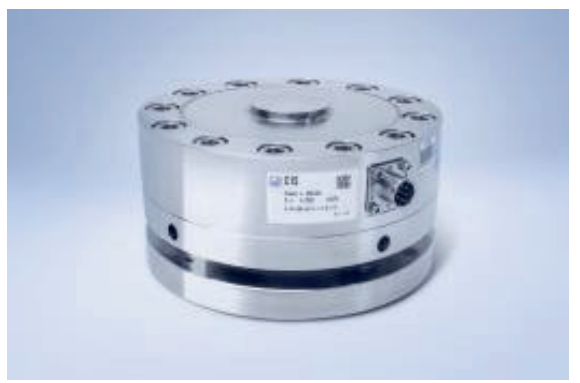

Transfernormal C15. Deutlich zu erkennen die Ringförmige Nut im Fußadapter, die Biegemomentsteifigkeit dieses Bauteils verringert. Der obere Messkörper ist durch Schrauben an dem Fußadapter montiert.

\section{References}

[1] Thomas Kleckers, Markus Graef, "New , IMEKO 22nd TC3, 12th TC5 and 3rd TC22 International Conferences; 3 to 5 February, 2014, Cape Town, Republic of South Africa

[2] Karl Hoffmann, "An introduction to Measurement using strain gauges", Hottinger Baldwin Messtechnik GmbH, 1989

[3] Thomas Kleckers, "Radial Shear Beams used as high precision transfer TRANSDUCERS", IMEKO 2010 TC3, TC5 and TC22 Conferences Metrology in Modern Context, November 22-25, 2010,

Pattaya, Chonburi, Thailand 